\title{
Improvement of Antiamoebic Activity of Rokitamycin Loaded in Chitosan Microspheres
}

\author{
Giovanna Rassu, Elisabetta Gavini, Antonella Mattana and Paolo Giunchedi ${ }^{*}$
}

Department of Drug Sciences, University of Sassari, Sassari, Italy

\begin{abstract}
Rokitamycin is a new macrolide containing 16 carbon atoms, strongly inhibitory for Acanthamoeba castellanii, an opportunistic protozoa of humans which cause primarily amoebic keratitis and chronic, but fatal, amoebic granulomatous meningoencephalitis. Chitosan microspheres were prepared as carriers to obtain a controlled release of rokitamycin, able to improve the antiamoebic activity of this drug. The microparticles were in vitro characterised and the efficacy of rokitamycin alone and encapsulated into microspheres on the growth rate of Acanthamoeba castellani was evaluated. The results obtained suggest that spray-drying is a good technique for the preparation of microspheres loaded with rokitamycin. The loading of the drug into the polymeric network leads to an increase in the dissolution rate compared to drug raw material and improves and prolongs the in vitro antiamoebic activity of the drug. Thus, microspheres based on rokitamycin could be used in the therapy of systemic and topical infections caused by Acanthamoeba.
\end{abstract}

Keywords: Rokitamycin, chitosan microspheres, spray-drying, antiamoebic activity, Acanthamoeba castellanii.

\section{INTRODUCTION}

Macrolide antibiotics comprise a large group of drugs characterized by large lactonic cycles with $12,14,15$, or 16 atoms to which sugars and/or amino sugars are bound. They have been extensively used for the treatment of bacterial infections, as they are able to interfere selectively with protein biosynthesis by binding to the 50S component of the prokaryotic ribosome. Macrolides have broad-spectrum activities against gram-positive and gram-negative bacteria, including actinomycetes and mycobacteria, as well as treponemes, mycoplasmas, chlamydiae, rickettsiae, and some protozoa [1-5]. Rokitamycin is a semisynthetic 16membered-ring macrolide of the leucomycin group [6]. Compared to other macrolides and antibiotics, the treatment with rokitamycin is not widespread, though it has been used for therapeutic means in both Europe and Japan [7]. Thus, rokitamycin is actually studied as alternative drug to the analogous macrolide antibiotics, such as erythromycin, against antibiotic-susceptible and or resistant bacteria [8,9].

Recently Mattana and co-workers (2004) showed that rokitamycin is strongly inhibitory, in vitro, for Acanthamoeba castellanii and that low doses block the cytopathic effect of $A$. castellanii against WKD cells derived from the human cornea [10]. The 14-membered-ring macrolide erythromycin, instead, exerts only a modest inhibitory effect on A. castellanii [10]. Other investigators have also reported that the 15 -membered-ring macrolide azithromycin is in vitro effective against pathogenic Acanthamoeba [11]. In addition, azithromycin as well as rokitamycin exert amoebistatic activities at their MICs. Furthermore, also azithromycin is able to inhibit the cytopathic effect caused by

*Address correspondence to this author at the Department of Drug Sciences, University of Sassari, via Muroni 23/A, 07100 Sassari, Italy; Tel: +39-079-

228754; Fax +39-079-228715; E-mail: pgiunc@uniss.it
Acanthamoeba trophozoites on different human target cells [10].

Acanthamoeba are small, ubiquitous, free-living amoebas that can exist as motile trophozoites and double-walled cysts $[12,13]$. Since the early 1960 s, Acanthamoeba has been recognized as an opportunistic human pathogen capable of causing infections in both immunocompetent and immunocompromised hosts $[14,15]$. In particular, Acanthamoeba spp. are the causative agents of granulomatous amebic encephalitis (GAE), a fatal disease of the central nervous system, and amoebic keratitis (AK), a painful sight-threatening disease of the eyes $[16,17]$. They have also been associated with cutaneous lesions, arthritis and sinusitis in AIDS patients and other immunocompromised individuals [18-20].

Eradication of these protozoa from the infection sites is difficult for the high levels of resistance of both Acanthamoeba trophozoites and cysts to the usual antiprotozoal drugs and because the medical therapy is often less effective against cysts than against trophozoites [21]. Acanthamoeba keratitis therapy has been updated with the introduction of combination of different drugs (cationic antiseptics, aromatic diamidines, chlorhexidine) [22-24] but, at present, no clinical trial has been attempted for Acanthamoeba systemic infections yet. The therapeutic treatment for GAE is the most problematic; this is due to the weak abilities of many antiamoebic agents to cross the blood-brain barrier (BBB) into the central nervous system (CNS). Therefore, further research is needed to exploit new therapeutic agents and strategies.

These results suggested that rokitamycin might have great potential for the treatment of topical and systemic Acanthamoeba infections. To hypothesize its use in the therapeutic treatment of GAE, nevertheless, additional studies on the pharmacokinetics of these macrolides are needed to clearly demonstrate if these compounds can cross the 
blood-brain barrier and enter the CNS, since no data on this are apparently available in literature.

On the basis of these considerations, the aim of this study was to assess the feasibility of using biodegradable chitosan microspheres as carriers for controlled release of rokitamycin, able to improve the antiamoebic activity of the drug. Rokitamycin loaded and blank chitosan microspheres were prepared. Under reproducible cultural conditions, the efficacy of rokitamycin alone and encapsulated into microspheres as well as blank microspheres on the growth rate of Acanthamoeba castellani was evaluated.

Chitosan was chosen because it is a biodegradable natural polymer, with great potential for pharmaceutical applications due to its biocompatibility, high charge density, nontoxicity, antimicrobial activity and mucoadhesion properties [25]. Chitosans have been used in the design of different types of drug carriers for various administration routes such as oral, ocular, buccal, nasal, transdermal, parenteral, vaginal. Chitosan dosage forms can be engineered into different shapes and geometries such as nanoparticles, microspheres, hydrogels, films, fibers, sponges and rods [25, 26, 27-32].

\section{MATERIALS AND METHODS}

\section{Materials}

Rokitamycin (Mw: 827.99 g/mol; Purity: 95.2\%) (RK) was a kind gift from Grunenthal Formenti Laboratories (Milan, Italy). Chitosan Chitoclear ${ }^{\circledR}$ TM 1283 (Mw: $282 \mathrm{kDa}$; Deacetylation: $96 \%$; Viscosity: $224 \mathrm{cP})(\mathrm{C})$ was kindly donated by Primex Ehf (Siglufjordur, Islanda). Acetic acid (99.8\%, Sigma-Aldrich, Milan, Italy), ethanol (95-96\%, R.P. Normapur, Prolabo, Paris, France), 2-propanol (Fluka, Schnelldorf, Germany) and dimethyl sulphoxide (Prolabo, Fontenay, France) (DMSO) were used. Ultrapure (MilliQ) water was produced by an ultrapure water system (Millipore, Tokyo, Japan). Acetonitrile Chromanorm ${ }^{\circledR}$ HPLC grade (Prolabo, Paris, France) and ammonium acetate (Puriss, Ph. Eur., Sigma-Aldrich, Steinheim, Germany) were employed.

All the reagents used are of analytical grade.

\section{Preparation of Spray-Dried Microspheres}

The preparation of microspheres was carried out using a spray-drying technique.

Rokitamycin loaded microspheres, RK-C 1 and RK-C 2, were prepared using 1:3 and 1:4 drug-to-polymer weight ratios, respectively. Feed solutions of $1 \%(\mathrm{w} / \mathrm{v})$ concentration were prepared dissolving $1 \mathrm{~g}$ of solid (C plus RK) in 100 $\mathrm{mL}$ mixture of ethanol-water-acetic acid, in the ratio 50-49-1 $(\mathrm{v} / \mathrm{v})$ by magnetic stirring.

Microspheres were made by spraying the feed solutions with a Spray-dryer Mini Büchi B-191 (Büchi LaboratoriumsTechnik AG, Flawil, Switzerland). The solutions, kept on magnetic stirring, were fed to a $0.7 \mathrm{~mm}$ nozzle with a peristaltic pump (15\%), atomised by the force of compressed air ( 2 bar and $4501 / \mathrm{h}$ ) and blown together with heated air $\left(115^{\circ} \mathrm{C}\right)$ to a chamber where the solvents evaporated. The aspirator was set at $90 \%$. The dry particles were then cooled down and collected in the high-performance cyclone.

Applying the same spraying parameters, blank microparticles based on chitosan $(\mathrm{Cb})$ were prepared as comparison.
The formulations obtained were put in a desiccator under vacuum for at least $24 \mathrm{~h}$ before their characterisation.

The weight of the powder produced was measured and the yield of production was calculated as the weight percentage of the final amount with respect to the initial amount of drug and polymer in the feed solutions.

\section{Determination of Drug Content and Encapsulation Effi- ciency}

An exact amount of RK-C 1 and RK-C 2 microspheres (5 $\mathrm{mg}$ ) was weighed and dissolved with $400 \mu \mathrm{L}$ DMSO. Then, $25 \mathrm{~mL}$ phosphate buffer $\mathrm{pH} 6.5$ was added and the solution was leaved on magnetic stirring for $10 \mathrm{~min}$. Afterwards, $1 \mathrm{ml}$ of solution was transferred to an Eppendorf and centrifuged for $10 \mathrm{~min}$ at $13,000 \mathrm{rpm}$. The supernatant was analysed with the HPLC method described below.

RK is a drug with poor water wettability and its solubility is dependent on the $\mathrm{pH}$ of the medium [33]. The solubility of the drug in phosphate buffer $\mathrm{pH} 6.5$ was previously determined [34].

Each formulation was analysed in triplicate, each replicate injected in duplicate.

Standard curves were made showing linearity in the range $0.25 \mathrm{mg} / \mathrm{L}$ to $70 \mathrm{mg} / \mathrm{L}\left(\mathrm{R}^{2}=0.999\right)$.

The amount of effectively encapsulated drug (real drug content) was calculated as the experimentally detected amount of RK in microspheres with respect to the theoretical amount of the drug used for the preparation of particles and expressed as percentage. The encapsulation efficiency was determined from the ratio between the real drug content and the theoretical and expressed in per cent.

\section{Particle Size and Morphological Analysis}

Particle size and particle size distribution of the microspheres were determined using Coulter Laser diffractometric equipment (Coulter LS 100Q, Beckman Coulter Particle Characterization, Miami, Florida, USA). 2-propanol was used as the dispersion medium for the microspheres. Analyses were performed at room temperature under gentle magnetic stirring of the suspension.

Microspheres $(3 \mathrm{mg}$ ) were dispersed in $1 \mathrm{~mL}$ dispersion medium and sonicated for 2 min before analysis.

The results reported are the averages of the replicates' averages. The average particle size of the microspheres was expressed as the mean volume-surface diameter, $d_{v s}(\mu \mathrm{m})$.

The morphology of the microspheres was studied by Scanning Electron Microscopy (SEM). Samples of microspheres were placed on double-sided tapes, which had previously been secured on aluminium stubs, and analysed after gold sputtering at $20 \mathrm{kV}$ acceleration voltages under an argon atmosphere. The images were acquired.

\section{In Vitro Drug Release}

In vitro rokitamycin release tests from microspheres were performed with a USP dissolution apparatus (Erweka DT 70, Erweka GmbH, Heusenstamm, Germany), using the rotating basket method (Apparatus 1) and phosphate buffer $\mathrm{pH} 6.5$ as dissolution medium $(400 \mathrm{~mL})$. The temperature was set at 37 $\pm 0.5^{\circ} \mathrm{C}$ with a rotational speed of $50 \pm 1 \mathrm{rpm}$. The sink con- 
ditions were assured. An amount of free drug or microspheres corresponding to $2 \mathrm{mg}$ of RK were used. At predetermined times $(5,10,15,20,25,30,45,60,90,120$ and 180 $\mathrm{min}), 1 \mathrm{~mL}$ samples were withdrawn and the sample volume was replaced with fresh phosphate buffer. The samples withdrawn were centrifuged for $10 \mathrm{~min}$ at $13,000 \mathrm{rpm}$ and the supernatant analysed.

The amount of rokitamycin released, at each time point, was quantified with the HPLC method below described. The tests were performed in triplicate

As comparison, dissolution rate of RK in the same conditions was determined.

\section{HPLC Method for the Determination of Rokitamycin}

The analytical HPLC method for the quantitative determination of rokitamycin is a modification of the method presented by Tod and co-workers (1992) [35].

Determination of RK was performed by a liquid chromatography system Varian ProStar 210 equipped with an AutoSampler 410 and a PDA photodiode array detector. The chromatographic separation of RK was performed using a Hypersil BDS C18, $5 \mu \mathrm{m}, 150 \times 4.6 \mathrm{~mm}$ (ID) stationary phase, coupled with a Hypersil C18 precolumn. The mobile phase consisted of acetonitrile and $0.05 \mathrm{M}$ ammonium acetate in the ratio $3-2(\mathrm{v} / \mathrm{v})$ at $1.5 \mathrm{~mL} / \mathrm{min}$ flow. The injection volume was $20 \mu \mathrm{L}$.

Rokitamycin was detected by UV at $230 \mathrm{~nm}$. The elution time was $10 \mathrm{~min}$ and the retention time of RK was about 5.7 min. All analyses were carried out at room temperature.

The interference of the polymers, phosphate buffer $\mathrm{pH}$ 6.5 and DMSO on the chromatograms was checked.

\section{Amoeba Cultivation}

Amoeba cultivation was performed using trophozoites of A. castellanii isolated from the corneal ulcer of a soft contact lens wearer (Marche Polytechnic University, Ancona, Italy) that were axenically grown at $25^{\circ} \mathrm{C}$ in peptone-yeast extractglucose (PYG) medium [36]. The species identification of this isolate was based on cyst morphology and indirect immunofluorescence microscopy. Before the experiments, universal bacterial primers were used to detect any bacteria that may have been present, and protozoa were tested by PCR [37] and were found to be free of any contamination. Amoebas used as inocula were taken from logarithmic-phase cultures. Incubation at $37^{\circ} \mathrm{C}$ was routinely adopted for drug testing, as previous observations have shown that this Acanthamoeba isolate is able to grow at this temperature.

\section{Evaluation of Cell Viability}

Cell viability was determined by staining the amoebas with nigrosin $(0.25 \% \mathrm{w} / \mathrm{v}$ in PBS buffer) and examining them in a hemacytometer (Nageotte chamber) with an inverted microscope (Zeiss, Tilaval 31) equipped with a $20 \times$ lens objective. In our experiments, trophozoites with viability higher than $95 \%$ were employed.

\section{Determination of Effects of Drug and Microspheres on Acanthamoeba Growth}

Rokitamycin was made up as stock solution containing $20 \mathrm{mg} / \mathrm{mL}$ in $95 \%(\mathrm{v} / \mathrm{v})$ ethanol. The solution was sterilized by filtration through a $0.22 \mu \mathrm{m}$ pore-size filter (Millex-GS; Millipore, Molsheim, France). Preliminary tests with ethanol were performed to ensure that no trophozoite inhibition occurred at the concentrations used.

Briefly, RK-C 1 microspheres, selected on the basis of in vitro characterization results, were dispersed in PYG medium at $75 \mu \mathrm{g} / \mathrm{mL}$ concentration of drug. Instead, drug-free microparticles were dispersed at $300 \mu \mathrm{g} / \mathrm{mL}$ : in such way, the effect of the highest amount of microspheres employed was tested. Experiments were performed in sterile 24-well plates (Corning). Serial twofold dilutions of the drug alone and of microsphere dispersions were prepared in PYG medium. Control wells received $1 \mathrm{~mL}$ of PYG medium in place of drug or microsphere dilutions. The amoebas, which were washed twice in phosphate-buffered saline $\mathrm{pH} 7.2$ (PBS), were suspended in PYG medium at a density of $8 \times 10^{3}$ cells $/ \mathrm{mL}$ or $1.6 \times 10^{4}$ cells $/ \mathrm{mL}$. One millilitre of the calibrated trophozoite suspension was added to each well, and then the plates were sealed and incubated at $37^{\circ} \mathrm{C}$ in a $5 \%$ $\mathrm{CO}_{2}$ atmosphere. Tests were performed in duplicate and were repeated at least three times. At selected time intervals (3, 4, 7, 9 and 15 days of incubation), amoebas growing in each well were counted in a Nageotte chamber, using the inverted microscope.

\section{Statistical Analysis}

Statistical differences between groups were determined using a two-tailed Student's t-test (GraphPad Prism, version 2.01; GraphPad software Incorporated). The difference was considered significant when $\mathrm{P}<0.05$.

\section{RESULTS}

\section{Preparation of Microspheres and Drug Content Deter- mination}

Spray-drying appears to be a suitable method to encapsulate RK in chitosan. The microspheres are obtained with good yields (50-54\%) and by a rapid time of production ( 5 $\mathrm{mL} / \mathrm{min}$ ). The loss of material could be explained, as previously reported, as a consequence of the relatively low volumes of feed solution sprayed for each batch of microspheres and by the structure of the spray-dryer apparatus, which is not equipped with a trap to recover the smallest and lightest particles which are exhausted by the aspirator [38].

The drug loaded quantification shows that the real drug content is close to theoretical value giving high encapsulation efficiencies (Table 1). RK-C 1 shows the highest yield of production and encapsulation efficiency.

Polymers do not interfere on the quantification of drug by HPLC analysis. The solubility of RK, in phosphate buffer $\mathrm{pH} 6.5$, results of $792.31 \mathrm{mg} / \mathrm{L}$.

\section{Particle Size and Morphological Analysis}

Size and size distribution of microparticles were measured by laser diffractometry. Data are listed in Table $\mathbf{1}$.

The encapsulation of RK into chitosan microspheres results in a smaller dvs compared to free $\mathrm{RK}$ and $\mathrm{Cb}$ formulation and in a change of particle size distribution: drug free microspheres are larger than loaded microparticles; it could be due to the higher viscosity of the feed solution of $\mathrm{Cb}$ 
Table 1. Characterisation of Spray-Dried Microspheres

\begin{tabular}{|c|c|c|c|c|c|}
\hline Formulations & RK to C Ratio (w/w) & Yield of Production (\%) & Real Drug Content (\%) ${ }^{a}$ & Encapsulation Efficiency (\%) ${ }^{a}$ & $d_{v s}(\mu m)^{a}$ \\
\hline RK-C 1 & $1-3$ & 53.6 & $20.57 \pm 1.65$ & $82.28 \pm 6.59$ & $2.69 \pm 0.15$ \\
\hline RK-C 2 & $1-4$ & 49.8 & $15.02 \pm 1.16$ & $74.60 \pm 5.78$ & $2.82 \pm 0.01$ \\
\hline $\mathrm{Cb}$ & $0-1$ & 53.4 & - & - & $5.72 \pm 0.12$ \\
\hline RK & - & - & - & - & $5.32 \pm 0.13$ \\
\hline
\end{tabular}

compared to RK-C 1 and RK-C 2 since the feed rate of polymeric solution can influence the particle size $[39,40]$.

When $\mathrm{Cb}$ particles are observed by SEM it would seem that microspheres are characterized by wide particle size distribution; however they show a Gaussian distribution curve when analysed by laser diffractometry. This can be explained with the use of ultrasounds for suspending the particles before laser diffraction analysis that could break the aggregates observed to the SEM.

\section{RK-C 1 and 2 Show Bimodal Curves}

$\mathrm{T}$ he morphology of the microspheres was examined using SEM. Microspheres produced show spherical shape and smooth surface (Fig. 1); only a few particles of $\mathrm{Cb}$ (Fig. 1C) have wrinkled surface.

No free drug crystals are found outside the particles confirming the effectiveness of RK encapsulation.

\section{In Vitro Drug Release}

In vitro release of rokitamycin from microspheres and the dissolution rate of pure drug were evaluated. Fig. (2) illustrates the results.

RK shows a slow dissolution rate: $6.5 \%$ of drug dissolves in the medium within $5 \mathrm{~min}$; the dissolution rate slightly increases and only $14 \%$ of RK is recovered in buffer after 180 $\min$.

As far as the formulations are concerned, they are characterized by different dissolution profiles during the first hour of the test $(\mathrm{P}<0.05)$ being the drug release rate from $\mathrm{RK}-\mathrm{C}$ 1 lower than RK-C 2 and only after 25 min a very small amount of drug is recovered in the dissolution medium. This can be due to the presence of an higher amount of chitosan in RK-C 2. It is known that chitosan and its salts are able to increase the solubility of poorly water-soluble drugs [41, 42, 29]. However after $1 \mathrm{~h}$, the RK release rate from RK-C 1 increases and no statistical differences are observed on the total amount of drug recovered in the dissolution medium at the end of the test which resulted of $14 \%$ and $27 \%$ in case of RK-C 1 and RK-C 2, respectively. Data not reported show that RK-C 1 release profile remarkably still increases and after $6 \mathrm{~h}$ the curves of formulations are almost superimposed while RK dissolution rate is still very slow. Thus the encapsulation of RK into chitosan microspheres determines an improvement of the dissolution rate of the drug with regard to the drug to polymer used. The different dissolution behavior between free RK and RK-C 1 and RK-C 2 along the time, can be attribute to the smaller particle size of the formulations compared to the free drug.

On the basis of these results and considering the higher yields of production and encapsulation efficiencies obtained in case of RK-C 1 compared with RK-C 2, the first formulation is selected for the in vitro microbiological study.

\section{Effects of Drug and Microspheres on Acanthamoeba Growth}

It has been previously showed that the lowest rokitamycin concentrations that cause complete $(100 \%)$ inhibition of A. castellanii growth (MIC100) at days 2, 3, and 6 are respectively $4.68 \mu \mathrm{g} / \mathrm{mL}, 18.75 \mu \mathrm{g} / \mathrm{mL}$ and $37.50 \mu \mathrm{g} / \mathrm{mL}$ and that the drug exerts amoebistatic activity at its MIC100 [10]. So, to evaluate the difference between the effect of free and encapsulated drug in chitosan microspheres on Acanthamoeba growth, in initial experiments RK was employed in dose equal to MIC100 at 3 days of incubation (18.75 $\mu \mathrm{g} / \mathrm{mL})$. The evaluation of Acanthamoeba growth curves shows that $8 \times 10^{3}$ trophozoites/well, after 15 days of incubation at $37^{\circ} \mathrm{C}$, in control cultures produce about $2.5 \times$ $10^{5}$ amoebas/well, with doubling times of about $72 \mathrm{~h}$. Under
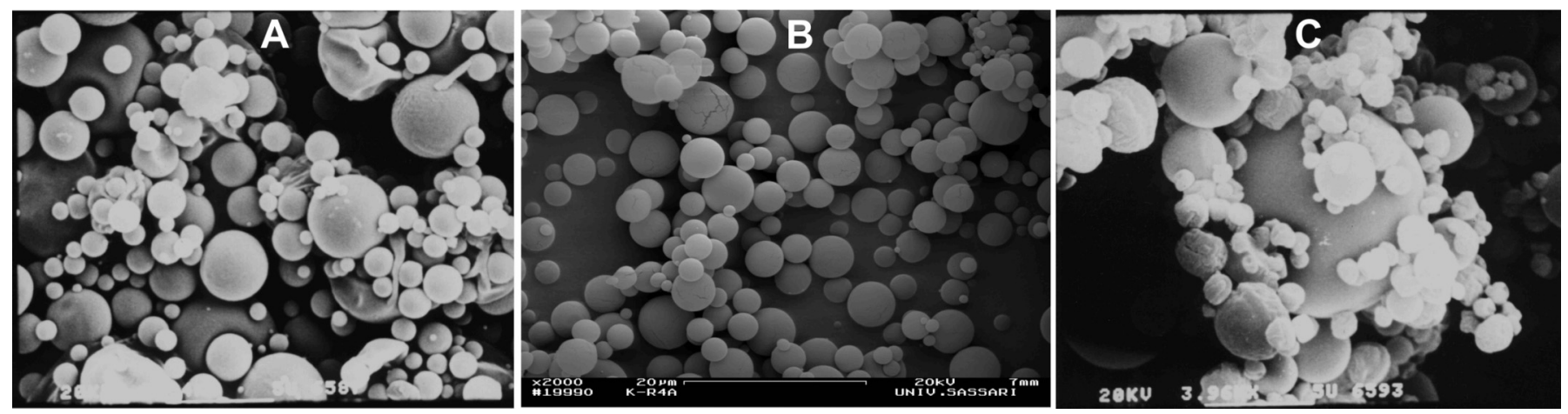

Fig. (1). SEM images of RK-C 1 (A), RK-C 2 (B) and Cb (C) spray-dried microparticles. Magnifications: 3840×(A), 2000× (B) and 3960× (C). 
the same experimental conditions (Fig. 3), chitosan microspheres, $\mathrm{Cb}$, do not alter the Acanthamoeba growth while the pure drug, RK, exerts amoebistatic activity that gradually decreases over time. On the contrary, the use of RK-C 1 microspheres causes complete antiamoebic activity until 15 days of incubation.

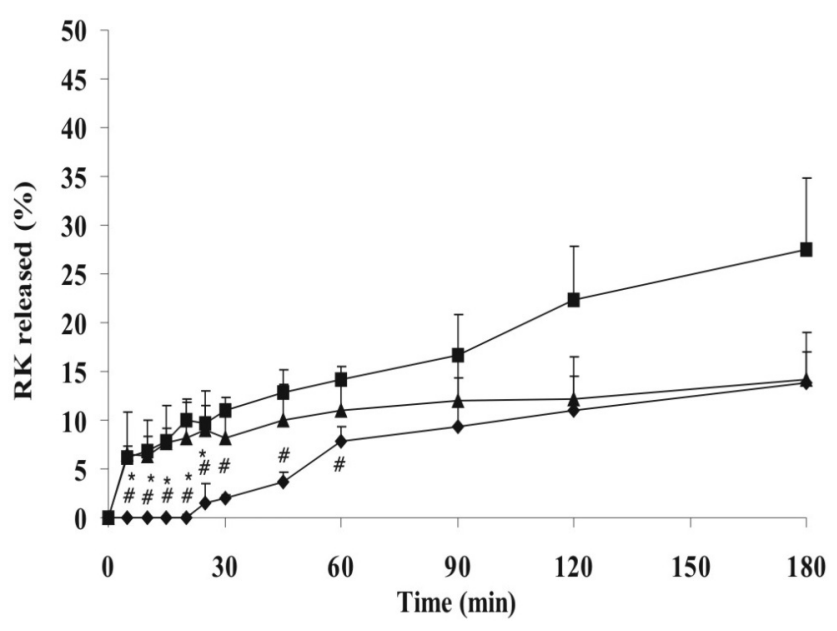

Fig. (2). In vitro release profiles of RK from RK-C 1 ( $\downarrow$ ) and RK-C 2 (घ) microspheres compared with the dissolution rate of pure RK $(\boldsymbol{\Delta})$; data are reported as means \pm SD (SD less then $4 \%$ ); $n=3$. Two-tailed t-test: \#: P $<0.05$ compared with RK-C 2 formulation; *: $\mathrm{P}<0.05$ compared with RK.

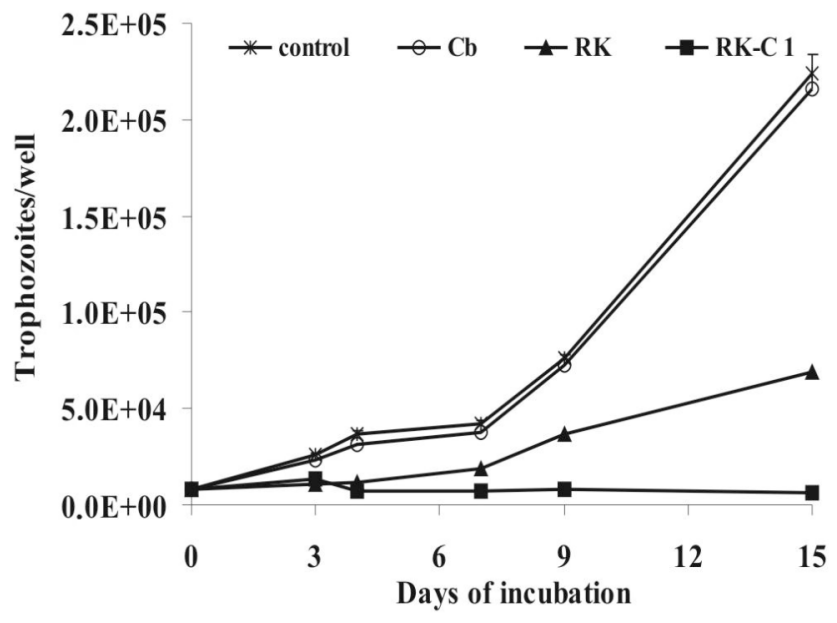

Fig. (3). Growth curves of $A$. castellanii suspended in PYG medium at a density of $8 \times 10^{3}$ trophozoites $/ \mathrm{ml}$ in the absence (control) or in the presence of drug, drug-loaded and free microspheres and cultured in sterile plates at $37^{\circ} \mathrm{C}$ in a $5 \% \mathrm{CO}_{2}$ atmosphere. $\mathrm{RK}$ was employed in dose of $18.75 \mu \mathrm{g} / \mathrm{mL}$ which corresponds to MIC100 at 3 days of incubation. Values are means \pm the standard errors (SE) of four experiments.

According to these results, the same test was carried out incubating $1.6 \times 10^{4} /$ well $A$. castellani trophozoites with different doses of RK $(9.37 \mu \mathrm{g} / \mathrm{mL}$ and $37.5 \mu \mathrm{g} / \mathrm{mL})$ in both free and encapsulated forms. In this case, after 15 days of incubation at $37^{\circ} \mathrm{C}$, control cultures produce about $4.86 \times$ $10^{5}$ amoebas/well, with doubling times of about $72 \mathrm{~h}$.
The growth curves, shown in Fig. (4), confirm that chitosan does not affect the trophozoites growth even if at 150 $\mu \mathrm{g} / \mathrm{mL}$ concentration and RK pure drug generate significant growth inhibition depending on dose and time exactly like the previous study $(\mathrm{P}<0.05)$.

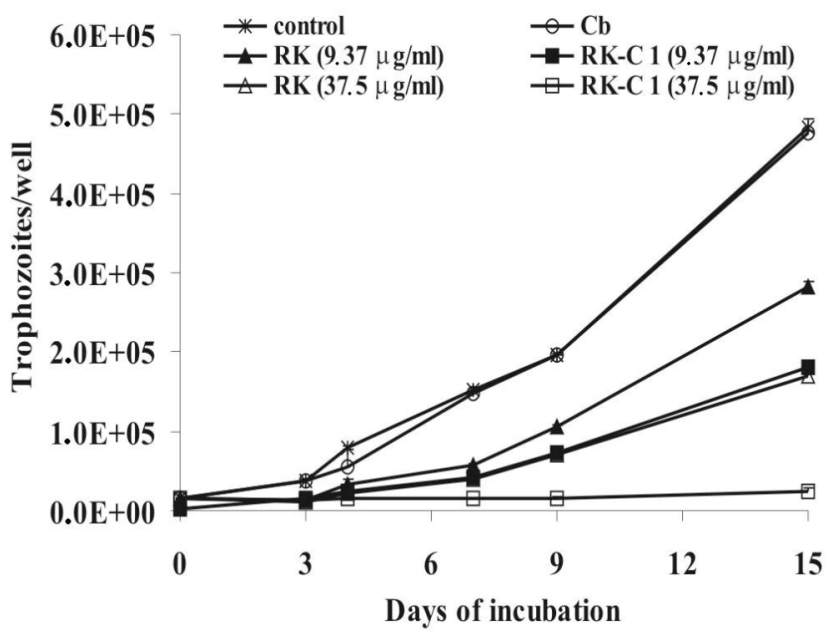

Fig. (4). Effects of drug and microspheres on Acanthamoeba growth using different drug concentrations $(9.37 \mu \mathrm{g} / \mathrm{mL}$ and 37.5 $\mu \mathrm{g} / \mathrm{mL}$ ); a density of $1.6 \pm 10^{4}$ trophozoites $/ \mathrm{ml}$ in the absence (control) or in the presence of drug, drug-loaded and free microspheres and cultured in sterile plates at $37^{\circ} \mathrm{C}$ in a $5 \% \mathrm{CO}_{2}$ atmosphere. Values are means $\pm S E ; n=6$.

Moreover, these growth curves demonstrate also that the antiamoebic activity of RK encapsulated in chitosan microspheres is dose- and time-dependent and that, up to 9 days there are not differences between RK and RK-C 1 used at the same concentration $(P>0.05)$. Nevertheless, from day 9 to day 15 of incubation both RK-C 1 concentrations (9.37 $\mu \mathrm{g} / \mathrm{mL}$ and $37.5 \mu \mathrm{g} / \mathrm{mL}$ ) show significantly greater inhibition capacity on Acanthamoeba castellanii growth than RK alone $(\mathrm{P}<0.05)$; besides, the growth curve of $9.37 \mu \mathrm{g} / \mathrm{mL}$ RK-C 1 is superimposable with that obtained using free RK at 37.5 $\mu \mathrm{g} / \mathrm{mL}$ concentration $(\mathrm{P}<0.05)$ (Fig. 4).

\section{CONCLUSIONS}

The findings obtained assessing the feasibility of using biodegradable chitosan microspheres as carriers for controlled rokitamycin-release, now suggest that the 16membered semisynthetic macrolide can be formulated into spray-dried microspheres. In addition they indicate that the loading of rokitamycin in the chitosan microspheres improves and prolongs the in vitro antiamoebic activity of the drug. Further in vitro and in vivo studies are in progress to analyze the susceptibilities of other pathogenic Acanthamoeba isolates to both free RK and RK-C, and to investigate the potential use of microspheres based on chitosan and its salts as carrier for ocular application for the therapy of keratitis as well as to target the drug to the brain by nasal administration as possible strategy for the therapeutic treatment of GAE. Nevertheless, data reported here suggest that microspheres based on rokitamycin could be used in the therapy of systemic and topical infections caused by Acanthamoeba. 


\section{ACKNOWLEDGEMENTS}

This work was supported by MiUR through grant PRIN05 and by Fondazione Banco di Sardegna. The authors thank "Farmaceutici Formenti S.p.A" for the donation of Rokitamycin.

\section{REFERENCES}

[1] Bryskier, A.; Labro, M.T. Presse Med., 1994, 23, 1762.

[2] Fichera, M.E.; Bhopale, M.K.; Ross, D.S. Antimicrob. Agents Chemother., 1995, 39, 1530.

[3] Georgopoulos, A.; Linnau, K.F.; Buxbaum, A.; Coste, C., Ramirez de Los Santos, M.A.; Shabpar, A.; Graninger, W.. Wien. Klin. Wochenschr., 2001, 113, 593.

[4] McKenna, S.; Evans, G.A. Can. J. Infect. Dis., 2001, 12, 218

[5] Goswick, S.M.; Brenner, G.M. Antimicrob. Agents Chemother., $\mathbf{2 0 0 3}, 47,524$

[6] Kanfer, I.; Skinner, M.F.; Walker, R.B. J. Chromatogr. A, 1998, $812,255$.

[7] Martindale: The Complete Drug Reference ${ }^{\circ} 2007$, The Pharmaceutical Press. https:/www.medicinescomplete.com $/ \mathrm{mc} / \mathrm{martindale}$ (accessed March 21 2007)

[8] Mazzariol, A.; Koncan, R.; Vitali, L.A.; Cornaglia, G. J. Antimicrob. Chemother., 2007, 59, 1171.

[9] Yano, H.; Kobayashi, T.; Okitsu, N.; Aoki, A.; Toriya, M.; Nakada, Y.; Sagai, H.; Iyobe, S.; Inoue, M. Jpn. J. Antibiot., 2007, 60, 31

[10] Mattana, A.; Biancu, G.; Alberti, L.; Accardo, A.; Delogu, G.; Fiori, P.L.; Cappuccinelli, P. Antimicrob. Agents Chemother., 2004, 48,4520 .

[11] Schuster, F.L.; Visvesvara, G.S. J. Eukaryot. Microbiol., 1998, 45, 612 .

[12] Ma, P.; Visvesvara, G.S.; Martinez, A.J.; Theodore, F.H.; Daggett, P.M.; Sawyer, T.K. Rev. Infect. Dis., 1990, 12, 490

[13] Saragoza, R. Crit. Rev. Microbiol., 1994, 20, 225.

[14] Ferrante, A. Parasite Immunol., 1991, 13, 31.

[15] Schuster, F.L.; Visvesvara, G.S. Int. J. Parasitol., 2004, 34, 1001.

[16] Marciano-Cabral, F.; Cabral, G. Clin. Microbiol. Rev., 2003, 16, 273 .

[17] Sharma, S.; Pasricha, G.; Das, D.; Aggarwal, R.K. Arch. Ophthal., 2004, 122, 1430 .

[18] Jeansson, S.; and Kvien, T.K. Scand. J. Immunol., 2001, 53, 610.

[19] Levine, S.; Goldstein, A.E.; Dahdouh, M.; Blank, P.; Hoffman, C.; Gropper, C.A. Cutis, 2001, 67, 377.
[20] Torno, M.S.; Babapour, R.; Gurevitch, A.; Witt, M.D. J. Am. Acad. Dermatol., 2000, 42, 351 .

[21] Lloyd, D.; Turner, N.A.; Khunkitti, W.; Hann, A.C.; Furr, J.R.; Russell, A.D. J. Eukaryot. Microbiol., 2001, 48, 11.

[22] Khunkitti, W.; Lloyd, D.; Furr, J.R.; Russell, A.D. J. Appl. Microbiol., 1997, 82, 107.

[23] Hargrave, S.L.; McCulley, J.P.; Husseini, Z. Ophthalmology, 1999 $106,952$.

[24] Kosrirukvongs, P.; Wanachiwanawin, D.; Visvesvara, G.S.; Ophthalmology, 1999, 106, 798.

[25] Singla, A.K.; Clawla, M. J. Pharm. Pharmacol., 2001, 53, 1047.

[26] Sinha, V.R.; Singla, A.K.; Wadhawan, S.; Kaushik, R.; Kumria, R.; Bansal, K.; Dhawan, S. Int. J. Pharm., 2004, 274, 1.

[27] Alonso, M.J.; Sanchez, A. J. Pharm. Pharmacol., 2003, 55, 145

[28] Gavini, E.; Rassu, G.; Sanna, V.; Cossu, M.; Giunchedi P. J. Pharm. Pharmacol., 2005, 57, 287.

[29] Denkbas, E.B. J. Bioact. Compat. Polym., 2006, 21, 351.

[30] Gavini, E.; Hegge, A.B.; Rassu, G.; Sanna, V.; Testa, C.; Pirisino, G.; Karlsen, J.; Giunchedi, P. Int. J. Pharm., 2006, 307, 9.

[31] Gavini, E.; Rassu, G.; Muzzarelli, C.; Cossu, M.; Giunchedi, P, Eur. J. Pharm. Biopharm., 2008, 68, 245.

[32] Dalpiaz, A.; Gavini, E.; Colombo, G.; Russo, P.; Bortolotti, F.; Ferraro, L.; Tanganelli, S.; Scatturin, A.; Menegatti, E.; Giunchedi, P. J. Pharm. Sci., 2008, DOI: 10.1002/jps 21335

[33] Petitjean, O.; Tod, M.; Louchahi, K. J. Pharm. Biomed. Anal. 1995, 13, 817 .

[34] Fini, A. In Principi di tecnologie farmaceutiche; Colombo, P.; Castellani, P.L.; Gazzaniga, A.; Menegatti, E.; Vidale, E., Eds.; Casa Editrice Ambrosiana: Milano, 2004; pp. 578-612.

[35] Tod, M.; Biarez, O.; Nicolas, P.; Petitjean, O. J. Chromatogr. 1992, 575, 171.

[36] Garcia, L.S.; Bruckner, D.A. Diagnostic medical parasitology, 2nd ed.; American Society for Microbiology: Washington, 1993; pp. 601-605.

[37] Kotilainen, P.; Jalava, J.; Meurman, O.; Lehtonen, O.P.; Rintala, E.; Seppiala, O.P.; Eerola, E.; Nikkari, S. J. Clin. Microbiol., 1998, $36,2205$.

[38] Giunchedi, P.; Gavini, E.; Bonacucina, G.; Palmieri, G.F. J. Microencapsul., 2000, 17, 711.

[39] Giunchedi, P.; Conte, U. S.T.P. Pharma Sci., 1995, 5, 276.

[40] Esposito, E.; Roncarati, R.; Cortesi, R.; Cervellati, F.; Nastruzzi, C Pharm. Dev. Technol., 2000, 5, 267.

[41] Portero, A.; Remuñán-López, C.; Vila-Jato, J.L. Int. J. Pharm., 1998, 175,75 .

[42] Mura, P.; Zerrouk, N.; Mennini, N.; Maestrelli, F.; Chemtob, C. Eur. J. Pharm. Sci., 2003, 19, 67. 